\title{
Relationship between Voided Urine Volume and Urinary ATP in Healthy Volunteers
}

\author{
Kimio Sugaya ${ }^{1,2 *}$, Saori Nishijima², Katsumi Kadekawa², Katsuhiro Ashitomi², \\ Hideyuki Yamamoto 3 \\ ${ }^{1}$ Department of Urology, Kitakami Central Hospital, Okinawa, Japan \\ ${ }^{2}$ Southern Knights' Laboratory, Okinawa, Japan \\ ${ }^{3}$ Department of Biochemistry, Graduate School of Medicine, University of the Ryukyus, Okinawa, Japan \\ Email: *sugaya@med.u-ryukyu.ac.jp
}

How to cite this paper: Sugaya, K., Nishijima, S., Kadekawa, K., Ashitomi, K. and Yamamoto, H. (2018) Relationship between Voided Urine Volume and Urinary ATP in Healthy Volunteers. Open Journal of Urology, 8, 275-280.

https://doi.org/10.4236/oju.2018.810031

Received: September 10, 2018

Accepted: October 13, 2018

Published: October 16, 2018

Copyright (c) 2018 by authors and Scientific Research Publishing Inc. This work is licensed under the Creative Commons Attribution International License (CC BY 4.0).

http://creativecommons.org/licenses/by/4.0/

\begin{abstract}
Purpose: We examined the relationship between the urinary ATP level and the desire to void, voided urine volume, and urinary osmotic pressure. Materials and Methods: The subjects were 4 healthy volunteers $(2$ men and 2 women) without micturition disorders who were not taking any medications and had normal urinalysis findings. Over 2 - 3 days, they urinated into a clean cup a total of 20 times. The strength of the desire to void was classified (none, slight, moderate, or strong; scored from 1 to 4 , respectively) and a voiding score was assigned at each urination, along with measurement of the voided volume, urinary ATP, urinary creatinine, and urinary osmotic pressure (one man). Results: Variation of the urinary ATP/creatinine ratio was large in both male and female subjects. The urinary ATP/creatinine ratio was lower in the 2 men compared with the 2 women. This ratio showed a significant negative correlation $\left(\mathrm{R}^{2}=0.1577\right.$ and 0.1673 , both $\left.\mathrm{p}<0.05\right)$ with the voided urine volume in the male subjects. However, there was no relationship between the urinary ATP/creatinine ratio and the voiding score or voided urine volume in the female subjects. Conclusions: The urinary ATP level may not be a clinically useful marker of bladder pathology. Both the present study and our previous studies identified a large sex difference in the urinary ATP/creatinine ratio, and this finding may be important when considering the causes of overactive bladder $(\mathrm{OAB})$ in women. The mechanism underlying the increase in urinary ATP in women should be investigated to develop new therapies for $\mathrm{OAB}$.
\end{abstract}

\section{Keywords}

Urinary ATP, Voided Urine Volume, Sex Difference 


\section{Introduction}

Adenosine triphosphate (ATP) is a major neurotransmitter in both the motor and sensory neurons of the peripheral nervous system [1] [2]. Recently, it has become accepted that epithelial tissues (including the urothelium) may have a role in mediation of sensation [3] [4]. Exposure to pressure, stretch, or hypo-osmotic stimulation leads to release of ATP from epithelial cells [3] [5]. Although it is difficult to prove that urinary ATP is mainly derived from the bladder epithelium, it has been demonstrated that release of ATP from cultured epithelial cells is increased in patients with interstitial cystitis [6]. Therefore, the urinary ATP level may be a potential marker of bladder pathology.

In our previous study using rats, the urinary ATP level was increased after stimulation of the bladder by infusion of acetic acid, while this increase in urinary ATP was blocked by adrenergic alpha-1 receptor antagonists or anti-muscarinic agents [7] [8]. In a study of healthy volunteers who urinated into a clean cup when the desire for micturition was felt, the urinary ATP/creatinine ratio was lower in males than in females [9]. In patients with benign prostatic hyperplasia (BPH), administration of an alpha-1 receptor antagonist decreased lower urinary tract symptoms (LUTS) and reduced the urinary ATP/creatinine ratio, with improvement of LUTS being greater in patients who had a high baseline urinary ATP level. Furthermore, in patients with overactive bladder (OAB), an anti-muscarinic agent improved LUTS and decreased the urinary ATP/creatinine ratio, and LUTS showed more improvement when the baseline urinary ATP level was elevated. Moreover, improvement of LUTS in patients with $\mathrm{BPH}$ or $\mathrm{OAB}$ by treatment with an alpha-1 receptor antagonist or anti-muscarinic agent was related to reduction of the urinary ATP/creatinine ratio [9]. Therefore, it is possible that urinary ATP could be measured as a marker of bladder pathology.

However, urinary ATP is also influenced by bacterial infection and renal dysfunction [10], so it is necessary to exclude such factors. Also, any stimulation of epithelial cells leads to the release of ATP [3] [5], so the voided urine volume or intravesical urine volume could influence ATP release from the bladder epithelium. Therefore, we examined the relationship between the urinary ATP level and the desire to void, the voided urine volume, and the urinary osmotic pressure.

\section{Subjects and Methods}

The subjects were 2 male and 2 female healthy volunteers aged $32-52$ years without micturition disorders who were not taking any medications. They were all staff members at our previous hospital (Ryukyu University Hospital). Subjects gave informed consent to the study and the protocol was approved by the University of the Ryukyus. In all 4 subjects, Body Mass Index was 19.1 - 25.0, hepatic and renal function was normal in blood biochemical test, urinalysis including urine sediment was normal and absence of residual urine after micturition 
was confirmed on ultrasonography.

Over 2 - 3 days in January 2008, the subjects urinated into a clean cup for a total of about 20 times and they rated the desire to void as follows: none (1 point), slight ( 2 points), moderate ( 3 points), or strong ( 4 points). This voiding score $(1-4)$ was determined at every urination, along with the voided urine volume, urinary ATP, urinary creatinine, and urinary osmotic pressure (one man). Urinary ATP was analyzed immediately by using an ATP Hygiene Kit HS (Bio Thema AB, Haninge, Sweden) and a Gene Light 55 (Microtec Nition, Funabashi, Japan). The urinary creatinine level and osmotic pressure were measured at SRL Inc. (Tokyo, Japan). ATP values were corrected by the urinary creatinine level (ATP/creatinine ratio). Then the relationship between the $\mathrm{ATP} /$ creatinine ratio and the voiding score, voided urine volume, and osmotic pressure was examined.

Results are reported as the mean \pm standard deviation (SD). Student's paired or unpaired $t$-test and regression analysis were used for statistical analyses, and $\mathrm{p}<0.05$ was considered to indicate significance.

\section{Results}

The urinary ATP/creatinine ratio showed wide variation in both the male and female subjects. This ratio was lower in the 2 men compared with the 2 women (Figure 1). In the male subjects, the urinary ATP/creatinine ratio showed a significant negative correlation $\left(\mathrm{R}^{2}=0.1577\right.$ and 0.1673 , both $\left.\mathrm{p}<0.05\right)$ with the voided urine volume, but not with the urinary osmotic pressure (Figure 2). There was also a negative correlation tendency between the urinary ATP/creatinine ratio and the voiding score, but it was not significant. In the female subjects, there was no relationship between the urinary ATP/creatinine ratio and the voiding score or voided urine volume.

\section{Discussion}

This study revealed that the urinary ATP/creatinine ratio was lower in male subjects compared with female subjects, while our previous study identified a sex difference in the urinary ATP level [9]. According to these two studies, the urinary ATP/creatinine ratio was about 4-fold higher in healthy female volunteers than in male volunteers and was also about 2-fold higher in female OAB patients than in male $\mathrm{BPH}$ patients. At present, this marked sex difference in the urinary ATP/creatinine ratio cannot be explained. When ATP is produced in the mitochondria, oxygen radicals are also created as a by-product and these radicals strongly induce symptoms of $\mathrm{OAB}$ [11] [12]. OAB is often idiopathic in females, while it is usually secondary to $\mathrm{BPH}$ or neurogenic bladder in males. Therefore, a factor that increases the release of ATP and oxygen radicals from the bladder epithelium may exist in females, and this putative factor may be one of the causes of OAB.

The present study revealed a significant negative correlation of the urinary 


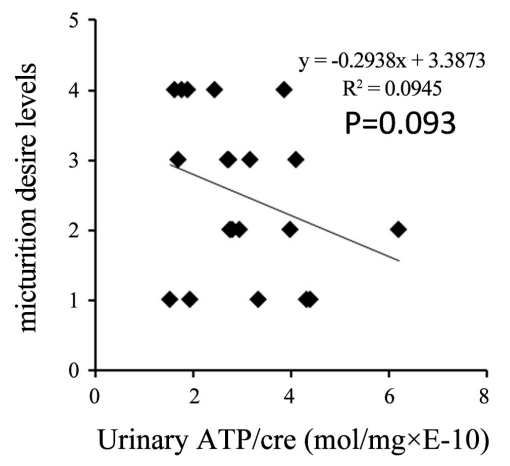

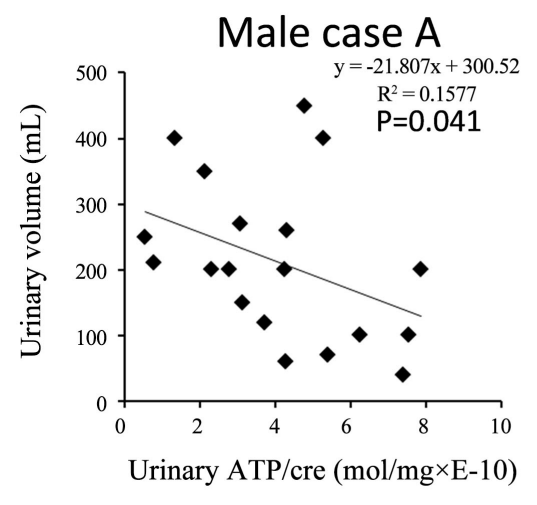

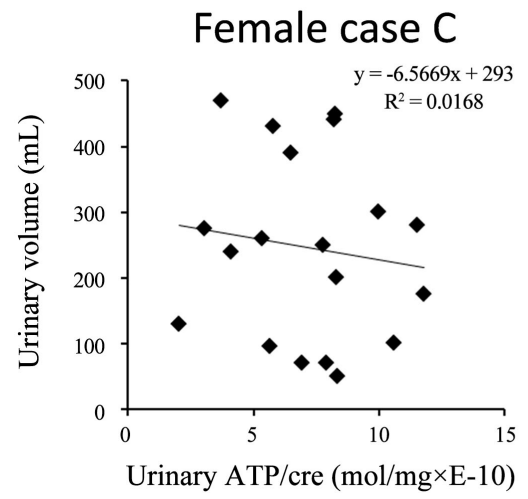

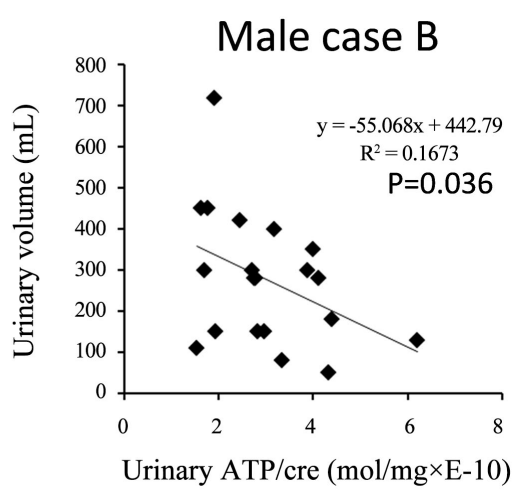

Female case D

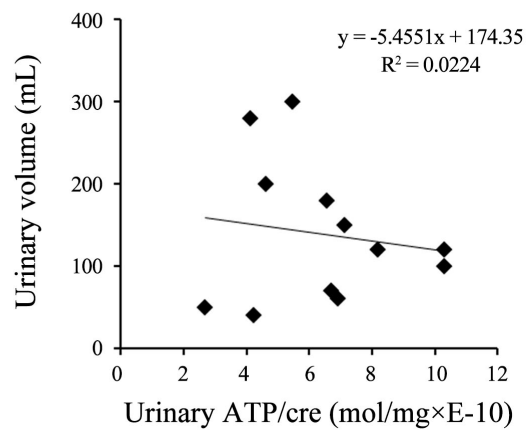

Figure 1. Relationship between the urinary ATP/creatinine ratio and the voided urine volume in 2 male volunteers (cases A and B) and 2 female volunteers (cases $\mathrm{C}$ and $\mathrm{D}$ ).
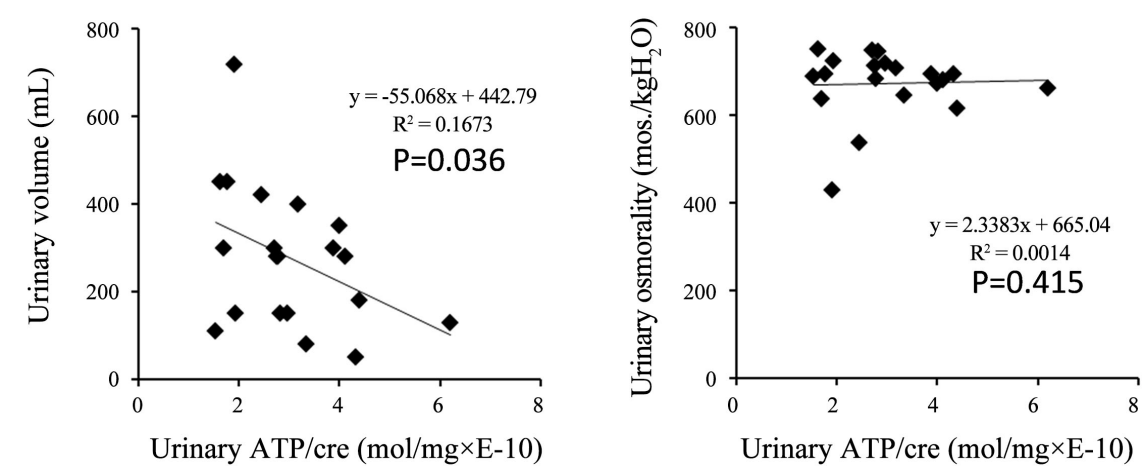

Figure 2. Relationship between the urinary ATP/creatinine ratio and the voiding score (a), voided urine volume (b), and urinary osmotic pressure (c) in case B from Figure 1 (a male volunteer).

$\mathrm{ATP} /$ creatinine ratio with the voided urine volume in the 2 male volunteers. Since exposure to pressure, stretch, or hypo-osmotic stimulation promotes the release of ATP from epithelial cells [3] [5], ATP secretion by the bladder epithelium may be high, not only when the bladder is full of urine, but also when the bladder is shrinking at the end of voiding. When the bladder is full, ATP secreted in response to pressure or stretch stimulation may be diluted by the large urine volume. Conversely, it is possible that ATP secreted as the bladder is shrinking at the end of voiding may not enter the urine. However, we found that the urinary ATP concentration was higher when the urine volume was smaller. 
In our male subjects, the urinary ATP/creatinine ratio was not correlated with the voiding score or the urinary osmotic pressure. This suggests that the intravesical urine volume and the desire to void are not necessarily related. Khera et al. reported that less ATP was released from the bladder by hypo-osmotic stimulation in normal rats than in rats with chronic spinal cord injury [5]. Therefore, urinary osmotic pressure may have a small influence on ATP secretion in healthy male volunteers. In our female subjects, the urinary ATP/creatinine ratio was not correlated with the voiding score or the voided urine volume. Release of ATP from the bladder epithelium was greater in females, and these parameters seemed to have little influence on ATP secretion.

It has been reported that the urinary ATP level is influenced by bacterial infection, renal dysfunction [10], voided urine volume, and gender. Therefore, the urinary ATP level may not be a useful marker of bladder pathology, although we previously reported that measurement of urinary ATP could be used clinically [9]. Interestingly, our research has identified a large sex difference in the urinary $\mathrm{ATP} /$ creatinine ratio, which may be an important finding when considering the etiology of $\mathrm{OAB}$ in females. In the present study, there were only two men and two women, and there is a limit to making more reliable results. Therefore, subjects should be increased in the next step, and the mechanism underlying the increase in ATP in females should be examined further in order to develop new treatments for $\mathrm{OAB}$.

\section{Conflicts of Interest}

The authors declare no conflicts of interest regarding the publication of this paper.

\section{References}

[1] Vizi, E.S., Liang, S.D., Sperlágh, B., Kittel, A. and Jurányi, Z. (1997) Studies on the Release and Extracellular Metabolism of Endogenous ATP in Rat Superior Cervical Ganglion: Support for Neurotransmitter Role of ATP. Neuroscience, 79, 893-903. https://doi.org/10.1016/S0306-4522(96)00658-6

[2] Andersson, K.-E. (2002) Bladder Activation: Afferent Mechanisms. Urology, 59, 43-50. https://doi.org/10.1016/S0090-4295(01)01637-5

[3] Ferguson, D.R. (1999) Urothelial Function. BJU International, 84, 235-242. https://doi.org/10.1046/j.1464-410x.1999.00187.x

[4] Birder, L.A., Nakamura, Y., Kiss, S., Nealen, M.L., Barrick, S., Kanai, A.J., Wang, E., Ruiz, G., De Groat, W.C., Apodaca, G., Watkins, S. and Caterina, M.J. (2002) Altered Urinary Bladder Function in Mice Lacking the Vanilloid Receptor TRPV1. Nature Neuroscience, 5, 856-860. https://doi.org/10.1038/nn902

[5] Khera, M., Somogyi, G.T., Kiss, S., Boone, T.B. and Smith, C.P. (2004) Botulinum Toxin A Inhibits ATP Release from Bladder Urothelium after Chronic Spinal Cord Injury. Neurochemistry International, 45, 987-993. https://doi.org/10.1016/j.neuint.2004.06.001

[6] Sun, Y., Keay, S., De Deyne, P.G. and Chai, T.C. (2001) Augmented Stretch Activated Adenosine Triphosphate Release from Bladder Uroepithelial Cells in Patients 
with Interstitial Cystitis. The Journal of Urology, 166, 1951-1956.

https://doi.org/10.1016/S0022-5347(05)65726-6

[7] Sugaya, K., Nishijima, S., Tasaki, S., Kadekawa, K., Miyazato, M. and Ogawa. Y. (2007) Effects of Propiverine and Naftopidil on the Urinary ATP Level and Bladder Activity after Bladder Stimulation in Rats. Neuroscience Letters, 429, 142-146. https://doi.org/10.1016/j.neulet.2007.10.003

[8] Nishijima, S., Sugaya, K., Kadekawa, K., Naka, H. and Miyazato, M. (2009) Comparison of the Effect of Anti-Muscarinic Agents on Bladder Activity, Urinary ATP Level, and Autonomic Nervous System in Rats. Biomedical Research (Tokyo, Japan), 30, 107-112.

[9] Sugaya, K., Nishijima, S., Kadekawa, K., Miyazato, M. and Mukouyama, H. (2009) Relationship between Lower Urinary Tract Symptoms and Urinary ATP in Patients with Benign Prostatic Hyperplasia or Overactive Bladder. Biomedical Research (Tokyo, Japan), 30, 287-294.

[10] Uehara, Y., Yanai, M. and Kumasaka, K. (2004) Evaluation of Renal Damage Using Urinary ATP Analysis. Nihon Jinzo Gakkai shi, 46, 693-699.

[11] Yu, H.-J., Chien, C.-T., Lai, Y.-J., Lai, M.-K., Chen, C.-F., Levin, R.M. and Hsu, S.-M. (2004) Hypoxia Preconditioning Attenuates Bladder Overdistension-Induced Oxidative Injury by Up-Regulation of Bcl-2 in the Rat. The Journal of Physiology, 554, 815-828. https://doi.org/10.1113/jphysiol.2003.056002

[12] Andersson, K.E., Nomiya, M., Sawada, N. and Yamaguchi, O. (2014) Pharmacological Treatment of Chronic Pelvic Ischemia. Therapeutic Advances in Urology, 6, 105-114. https://doi.org/10.1177/1756287214526768 